\title{
Development of an Optimization Framework for the Comparative Evaluation of the Ecoimpact of Powertrain Concepts
}

\author{
Arved Esser, Jean-Eric Schleiffer, Tobias Eichenlaub, \\ Stephan Rinderknecht, \\ Technical University Darmstadt
}

\section{Zusammenfassung}

Der Stand der Technik in der Bewertung der lebenswegbezogenen Treibhausgasemissionen verschiedener Antriebsstrangkonzepte basiert auf der Bewertung von Eigenschaften bestehender Fahrzeuge am Markt. Diese Fahrzeuge wurden allerdings mit unterschiedlichen Zielen entwickelt und mit diversen Nutzungsprofilen betrieben. Wie die Antriebsstrangkonzepte im Vergleich abschneiden würden, wenn sie optimal auf das jeweilige Untersuchungsszenario ausgelegt würden, bleibt unklar. Die Kenntnis über diese Optimalparametrierung der Antriebskonzepte ist jedoch nötig, um einen fairen Vergleich der Technologien zu ermöglichen. Dies gilt insbesondere dann, wenn innovative Antriebsstrangkonzepte sowie zukünftige Szenarien betrachtet werden, in denen deutliche Abweichungen zu heute üblichen Parametrierungen von Fahrzeugen möglich sind. Im Rahmen des öffentlich geförderten Projekts „FahrKLang“ wurde eine Optimierungsumgebung für die vergleichende Bewertung des „Real Ecological Impact“ (REI) verschiedener Antriebsstrangkonzepte entwickelt, die in diesem Beitrag vorgestellt wird. Für jedes Untersuchungsszenario werden optimale Antriebsparametrierungen der verschiedenen Antriebskonzepte zur Minimierung des REI und eine angepasste Betriebsstrategie ermittelt, wodurch eine einheitliche Bewertungsbasis geschaffen wird. Repräsentative Fahrzyklen ermöglichen dabei eine effiziente Bewertung der Konzepte auf Basis von realen Flottenfahrdaten innerhalb der Optimierungsumgebung.

\begin{abstract}
The state of the art in the evaluation of the ecological impact of various powertrain concepts is based on the evaluation of characteristics of existing vehicles on the market. However, these vehicles were developed with different objectives and are operated with various usage profiles. The potential of the powertrain concepts, if they were optimally designed for a given parameter
\end{abstract}


set of a scenario to be investigated, remains unclear. But the knowledge of these optimal parametrizations of the powertrain concepts is necessary to enable an unbiased comparison of the technologies, especially if future scenarios are considered in which significant deviations from current parameter sets are possible. This work is based on results of the publicly funded FahrKLang project, in which an optimization framework for the comparative assessment of the "Real Ecological Impact" (REI) of various powertrain concepts has been developed. For each investigated scenario, optimal powertrain parametrizations of the various concepts to minimize the REI and a customized operational strategy are identified, thereby creating an equal evaluation basis. Representative driving cycles enable an efficient evaluation of the concepts based on real fleet driving data within the optimization environment.

\section{Introduction and Motivation}

In order to determine the environmental impact of vehicle powertrain concepts, greenhouse gas emissions over the entire life cycle of a vehicle must be estimated. The vehicle production, its operation including the necessary energy supply as well as the end-of-life have to be considered. Current life cycle assessment studies use the parameters of vehicles that are already available on the market. For each powertrain concept to be investigated, characteristic component properties are assigned. Battery capacities and power ratings of energy converters are assigned to characteristic vehicles that are intended to represent whole classes of vehicles. Consumption values are often only taken into account in the form of estimations of average consumption and do not consider real driving profiles or the specific efficiency advantages of innovative concepts.

However, this approach must be questioned when the actual potential of innovative powertrain concepts to reduce greenhouse gas emissions is to be determined. The properties of vehicles already available on the market, which serve as a basis for the characteristic vehicles, were developed with different and very diverse objectives. It is not known to what extent the environmental impact of currently available vehicles has been taken into account in the design process. It remains unclear how different concepts would comparatively perform if they were optimally adapted to real driving profiles with the aim of minimizing greenhouse gas emissions. In this contribution, the results of the publicly funded project "FahrKLang" are presented, in which an overall optimization environment for the comparative assessment of the Real Ecological Impact (REI) of powertrain concepts was developed.

After an overview of the optimization framework in Section 2, which describes the approach in more detail, descriptions of the central principles of the comparative evaluation of the REI of 
concepts follow. These include the determination of representative driving cycles from large sets of real driving data from a fleet of vehicles in Section 3 as well as the determination of key performance indicators in the operational phase by simulation in Section 4. Section 5 shows the comparative evaluation of the optimal parametrizations of different powertrain concepts determined on the basis of different evaluation scenarios. In Section 6, we give an outlook on the extent to which the approach presented and the results achieved can contribute to the development of future, innovative powertrains.

\section{Optimization Framework}

The dimensioning and parametrization of powertrain components has a significant impact on the evaluation of the REI of vehicles. In order to be able to compare different powertrain concepts for a specific vehicle in the sense of a benchmark, the interactions between the individual components, the impact of the users behavior on powertrain efficiency as well as sizing impacts on the different phases of the lifecycle must be taken into account. An accurate dimensioning and parametrization with the aid of a holistic overall system optimization approach is needed.

Basis for the dimensioning of the components of powertrain concepts to be compared is the evaluation of key parameters of the operational phase like consumption using real driving data from a fleet of vehicles, which are intended to represent the characteristic driving behavior of users in Germany. A cycle synthesis method, based on [1] and further described in Section 3, generates representative driving cycles. The extensive data sets are thereby reduced to a necessary minimum. This enables a computationally efficient evaluation of powertrain concepts with the aid of simulation based on real driving data.

An optimization framework was developed to determine the optimal powertrain parametrization. To achieve this, an operating strategy based on a locally optimal control approach, called Equivalent Consumption Minimization Strategy (ECMS) [2, 3], is adapted for each variant. The compressed but representative driving cycles enable the investigation of the operational phase of the necessary multitude of variants with high computing efficiency. The objective function of the optimization, which is evaluated using a genetic algorithm, is not only made up of the greenhouse gas emissions during the operational phase, but is considering the entire life cycle. The production phase, in particular, is strongly influenced by the dimensioning of the components. Fig. 1 shows the framework of the optimization environment. For each powertrain concept, the minimum greenhouse gas emissions are determined based on a representative driving cycle by an optimal dimensioning and parametrization of the respective components. In 
this way, a reliable and unbiased basis for the comparison of different powertrain concepts is created.

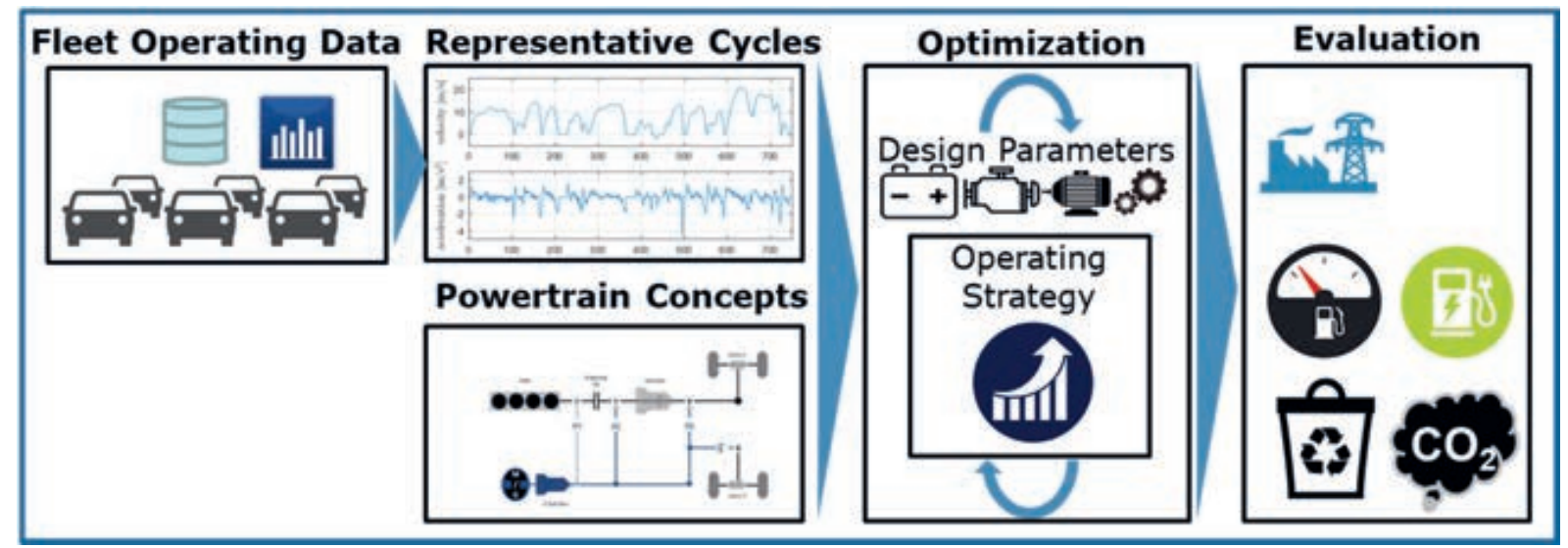

Fig. 1: FahrKLang Optimization Framework

The plausibility of the resulting vehicle parameters during the optimization process, which are set by a genetic algorithm by selection, mutation and recombination of the design parameters according to Table 1, is ensured by additional technology-neutral design constraints. The generated vehicles must be able to achieve a launch acceleration of $2,5 \mathrm{~m} / \mathrm{s}^{2}$ on a slope of $30 \%$, accelerate to $60 \mathrm{~km} / \mathrm{h}$ in 8 seconds and achieve a remaining acceleration of $0,33 \mathrm{~m} / \mathrm{s}^{2}$ at $180 \mathrm{~km} / \mathrm{h}$. Furthermore, all parametrizations of powertrain concepts that are capable of purely electric driving must achieve a purely electrical range of $60 \mathrm{~km}$ and a total range which is specified in a seperate table for each scenario showing the applied parameter sets. All powertrain concept parametrizations, presented in the results, fulfill these design constraints.

Table 1: Design parameters of the optimization process

\begin{tabular}{|l|l|}
\hline$N_{\mathrm{ICE}}$ & Index of the internal combustion engine in the database \\
\hline$P_{\mathrm{EM}}$ & Peak power of the electric machine \\
\hline$C_{\mathrm{Batt}}$ & Capacity of the battery \\
\hline$n_{\mathrm{Transm}}$ & Number of speeds in the transmission system \\
\hline$i_{\mathrm{N}}$ & Gear ratio of the transmission speeds $i_{1}$ to $i_{\mathrm{N}}$ \\
\hline$i_{\mathrm{EM}, \text { Hybrid }}$ & $\begin{array}{l}\text { Relative ratio of the connection of the electric machine for Parallel-Hy- } \\
\text { brid-Electric-Powertrains }\end{array}$ \\
\hline
\end{tabular}


Based on the calculated energy demand in the operational phase as well as the masses of components resulting from the powertrain dimensioning and parametrization, equivalent $\mathrm{CO}_{2}$ emissions over the entire life-cycle are finally determined with the help of researched emission factors for different scenarios to be investigated. The determined $\mathrm{CO}_{2}$ emissions in the production of the vehicles are shown in Fig. 7 for an exemplary scenario.

In this contribution, three scenarios are examined and discussed in detail in Section 5, which can be described with a set of parameters for each scenario (Table 4,

Table 7). In addition to evaluating the effects of changes in the total distance to be travelled without the need for a vehicle to be charged (scenario with and without the requirement for long-range capability), the effects of external factors such as emission factors for energy production or the energy density of energy storage systems are also examined by means of estimates for future enhancements (reference year 2018 vs. reference year 2030).

\section{Evaluation of Fleet Driving Data}

To enable a proper estimation of the REI of different powertrain concepts, real fleet driving data that depict the characteristic vehicle usage must be used as evaluation basis. Within the FahrKLang project, vehicles with the requirement of long-range capability were the main subject of research. Firstly, a detailed depiction of the driving behaviour concerning velocity and acceleration is required. Secondly, more general information about the distribution of trip distances and the total achieved mileage must be determined to enable a profound REI estimation.

Until now, there is no available data set containing extensive fleet driving data within Germany which was recorded over a longer period. Such a database would be necessary for a detailed depiction of the real usage behaviour and a real-driving based evaluation of the REI. As an estimation of this real fleet driving data, a data set that resulted from the "simTD" project [4] was used in the project, which contains recorded vehicle operation data on a $1 \mathrm{~Hz}$ basis from the metropolitan region of Frankfurt. The fleet consisted of different vehicles from different manufacturers that were driving in the regular traffic. The processed data set contains a total of 143.078 driven kilometres. The occurrence frequency of operating states as a function of velocity and acceleration and possible transitions between operating states are shown in Fig. 2. 


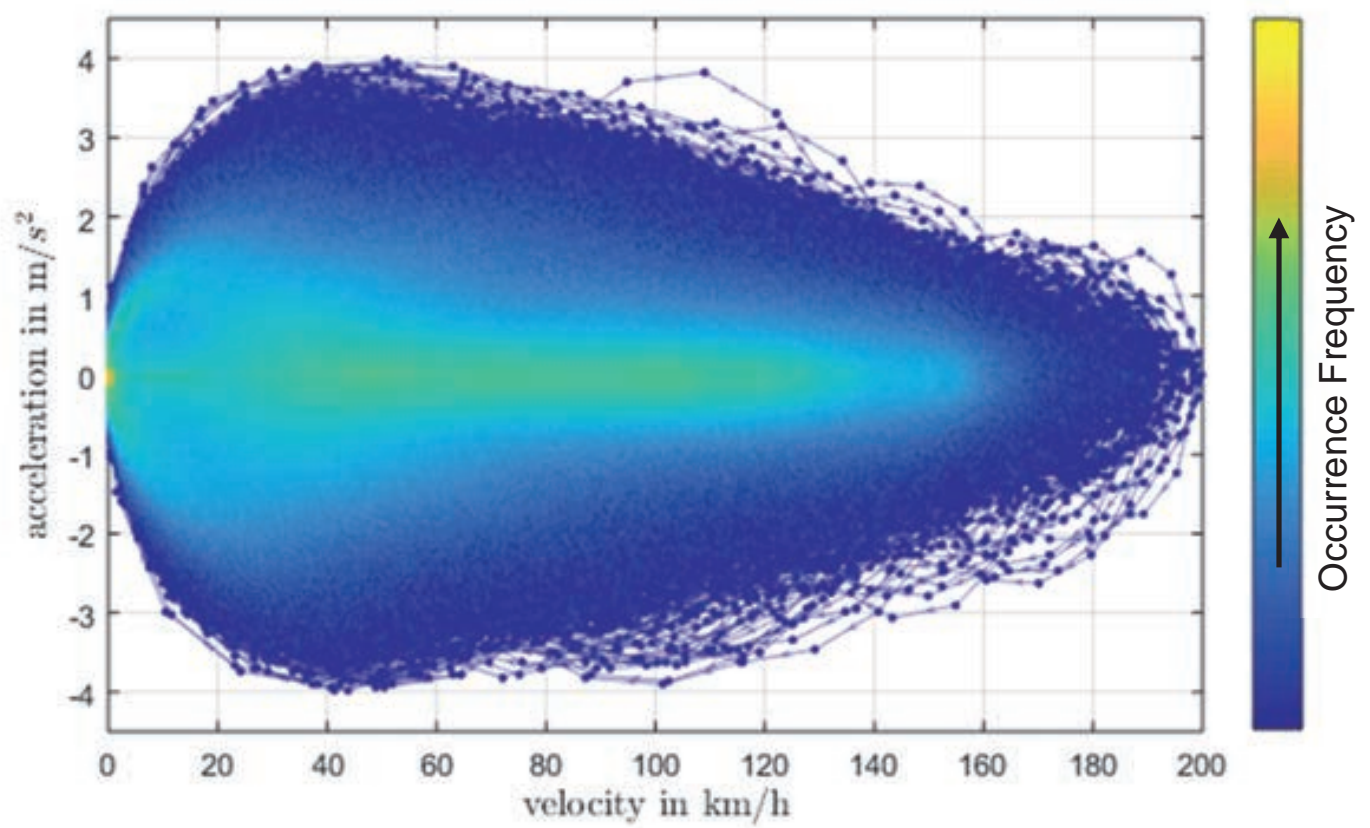

Fig. 2: Occurrence frequency of operating states of the fleet driving data as a function of velocity and acceleration. Nodes represent operating states while connections represent occurred transitions between the states.

Most frequent states are idling and cruising at constant speeds of $50 \mathrm{~km} / \mathrm{h}$ and $80-120 \mathrm{~km} / \mathrm{h}$. However, the available database lacks trips with large distances travelled at once, which is why the overall driving profile from Fig. 2 was considered for the scenarios with and without the requirement for long-range capability. The occurrence frequency shows that the largest part of the driving is at moderate speeds and accelerations, but less frequent events occure at high power demands or high decelerations.

For the simulation-based evaluation of different powertrain concept parametrizations within the optimization framework, representative driving cycles of the overall fleet driving profile are required. The stochastic cycle synthesis procedure proposed in [1] is used within this study. A driving cycle is assumed to be representative if it shows the same characteristic values of the criteria set $\boldsymbol{\theta}$ as the overall fleet profile shown in Fig. 2. The criteria set $\boldsymbol{\theta}$ is composed out of six single criteria $\theta_{1}, \theta_{2}, \ldots, \theta_{6}$, where $v$ is the velocity, $a$ is the longitudinal acceleration and $s$ is the travelled distance. The label "acc" indicates time periods of positive and zero traction forces, while "tot" stands for the total time period. 


$$
\begin{gathered}
\theta_{1}=\frac{1}{s_{\mathrm{tot}}} \int_{t \in \tau_{\mathrm{acc}}}^{t_{\mathrm{end}}} v(t)^{3} d t \\
\theta_{2}=\frac{1}{s_{\mathrm{tot}}} \int_{t \in \tau_{\mathrm{acc}}}^{t_{\mathrm{end}}} v(t) d t=\frac{s_{\mathrm{acc}}}{s_{\mathrm{tot}}} \\
\theta_{3}=\frac{1}{s_{\mathrm{tot}}} \int_{t \in \tau_{\mathrm{acc}}}^{t_{\mathrm{end}}} a(t) v(t) m d t
\end{gathered}
$$

$$
\theta_{4}=\frac{1}{t_{\text {end }}} \int_{0}^{t_{\text {end }}} v(t) d t
$$$$
\theta_{5}=\frac{1}{t_{\text {end }}} \int_{0}^{t_{\text {end }}}\left(v(t)-\theta_{4}\right)^{2} d t
$$

The relative error values are calculated for each criteria and the overall mean error is used as a final performance value to evaluate the quality of a driving cycle. Table 2 shows the exemplary calculation of the performance value for the first of the three chosen synthesized driving cycles, which can be seen in Fig. 4.

Table 2: Exemplary calculation of the performance value for the first of the three chosen synthesized driving cycles with a final performance value of $1,22 \%$.

\begin{tabular}{|c|c|c|c|c|c|c|c|}
\hline Criteria & $\begin{array}{c}\text { Single } \\
\text { cycle } \\
\text { value }\end{array}$ & $\begin{array}{c}\text { Fleet } \\
\text { data } \\
\text { value }\end{array}$ & Rel. error & Criteria & $\begin{array}{c}\text { Single } \\
\text { cycle } \\
\text { value }\end{array}$ & $\begin{array}{c}\text { Fleet } \\
\text { data } \\
\text { value }\end{array}$ & Rel. error \\
\hline$\theta_{1}$ & 338,2156 & 339,6601 & $0,43 \%$ & $\theta_{4}$ & 15,7919 & 15,3205 & $3,08 \%$ \\
\hline$\theta_{2}$ & 0,5316 & 0,5223 & $1,77 \%$ & $\theta_{5}$ & 129,6887 & 128,7599 & $0,72 \%$ \\
\hline$\theta_{3}$ & 0,1749 & 0,1734 & $0,84 \%$ & $\theta_{6}$ & 0,3151 & 0,3136 & $0,47 \%$ \\
\hline $\boldsymbol{\theta}$ & \multicolumn{7}{|c|}{} \\
\hline
\end{tabular}

The driving cycles should be of a minimum duration since an efficient evaluation of many parametrizations of a powertrain concept is necessary within the optimization framework and the computational effort scales approximately linear with the cycle duration. At the same time, it is more difficult to satisfy all criteria in the criteria set $\boldsymbol{\theta}$, when the duration of a cycle becomes shorter. For this reason, a parameter study was performed to identify a suited cycle duration. The results are shown in Fig. 3. For all investigated cycle durations from 300 s to 1500 s, 2000 driving cycles have been synthesized to create a pareto front showing the achievable performance value for each cycle duration. The graph shows that the best cycle quality improves significantly with the cycle duration at lower durations, while the quality increases rather slowly for higher cycle durations. A cycle duration of $800 \mathrm{~s}$ is chosen for the final evaluation, since 
further improvements of the performance value would require a significantly higher cycle duration.

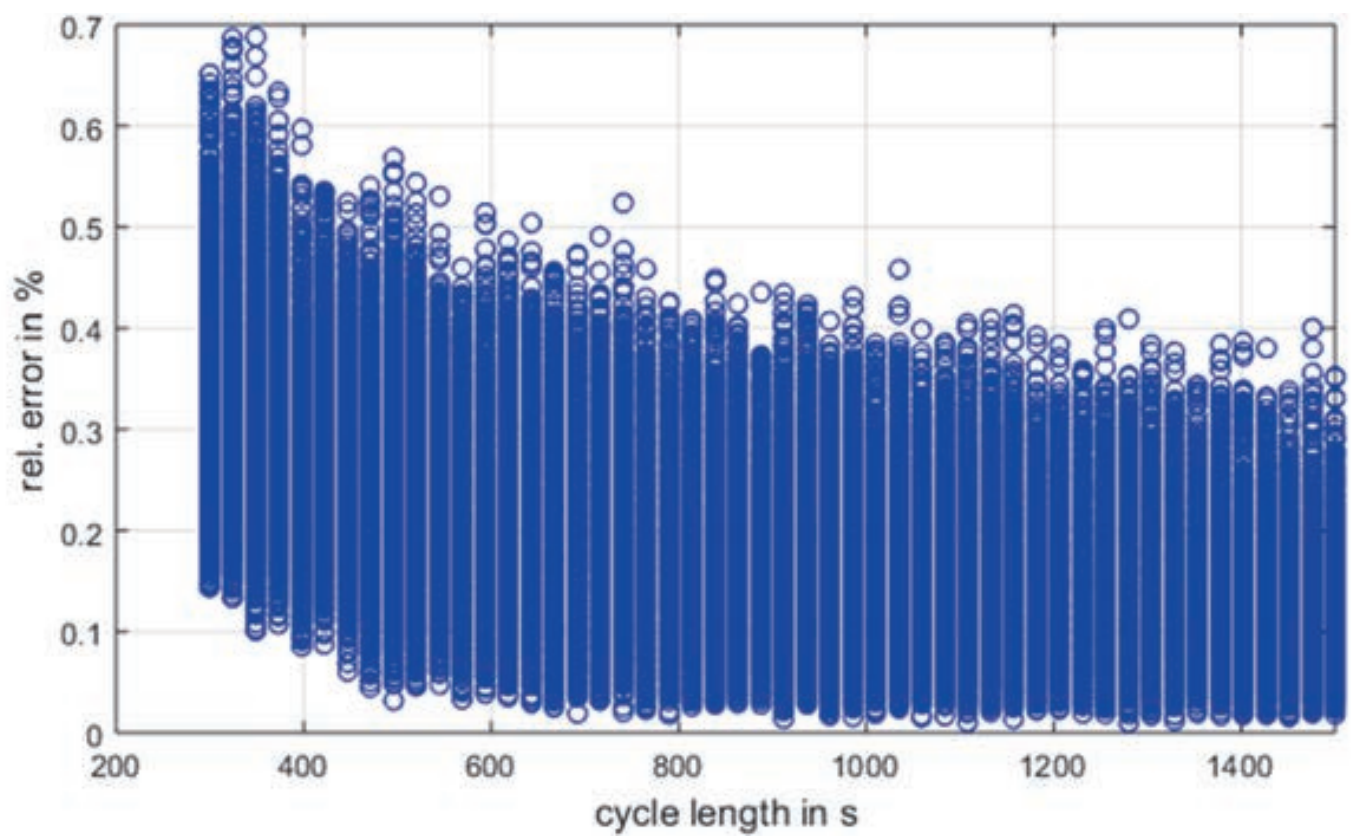

Fig. 3: Parameter study for the identification of required cycle duration. The achieveable performance value decreases with higher cycle durations.

After the final cycle duration has been determined, a more detailed study with the fixed duration of $800 \mathrm{~s}$ is performed. 50.000 cycles are synthesized and the resulting error distribution is shown in Fig. 4. The best cycles, shown on the right, have an error of 1-1,5\%. Regarding other uncertainties in the evaluation of the REI for different powertrain concepts, this error is considered as acceptable. Therefore using these representative driving cycles of Fig. 4 within the optimization framework enables an estimation of the REI based on the real driving profile shown in Fig. 2.

The second type of required information about trip distance distribution and mean vehicle mileage is retrieved from calculations of the DLR-VF, based on [5], that were also used in the FahrKlang project. From this source, the mean vehicle life mileage is assumed to be $158.000 \mathrm{~km}$, based on the distribution of achieved mileage for a compact class vehicle.

The trip distance distribution is especially relevant for Plug-in Hybrid Electric Vehicles (PHEV). On short trips, an almost purely electrical driving is possible while the battery capacity might be insufficient for higher distances, which is why the combustion engine has to be used more often with increasing trip distances. For a proper estimation of the consumptions of PHEV 
powertrain concepts, different trip distances have to be taken into account for the simulation. The final consumption values are then computed by weighting the results with the real occurrence frequency of the different trip distances.
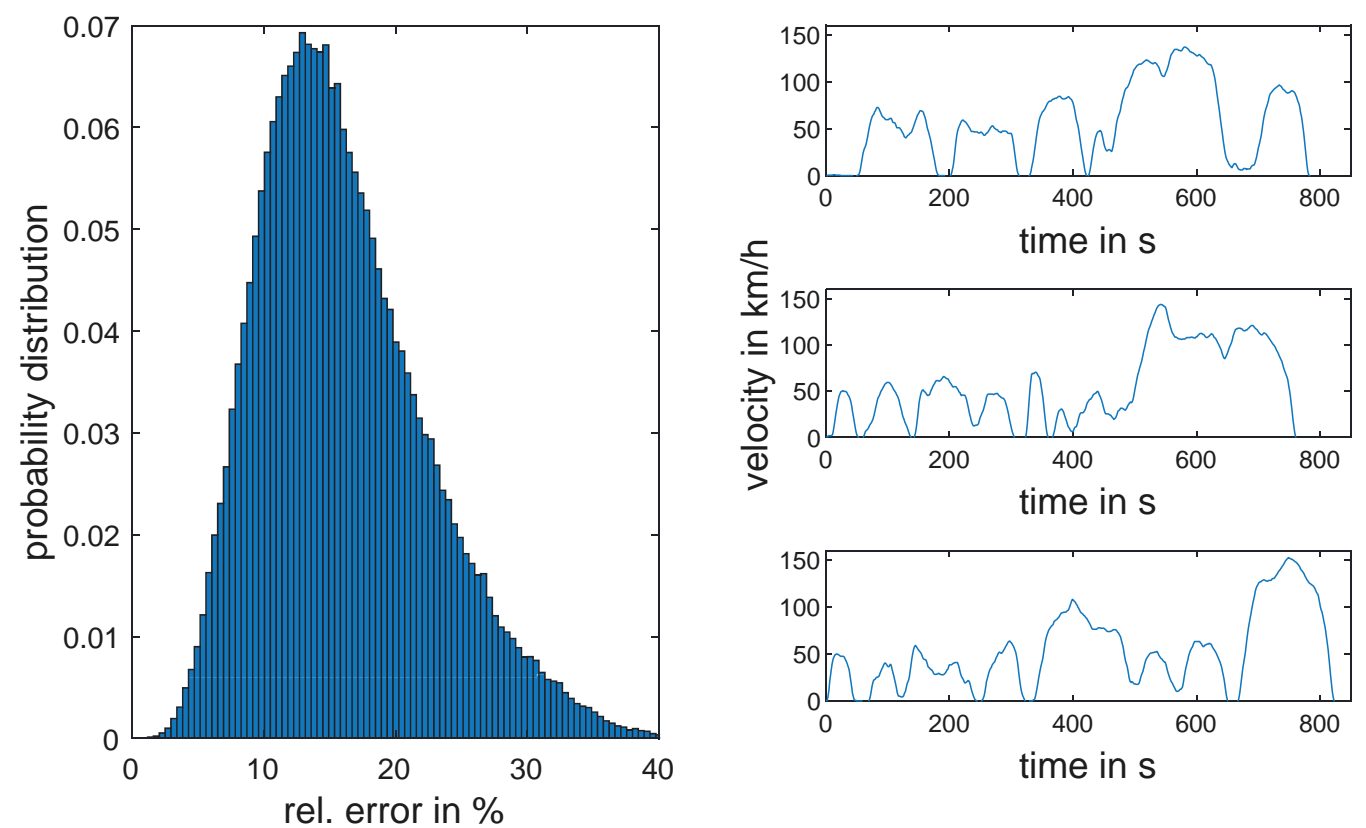

Fig. 4: Error distribution of the 50.000 synthesized cycles and the three chosen cycles.

For the scenarios with the requirement for long-range capability, which were the main investigation focus, trip distances above $100 \mathrm{~km}$ were considered. Three distances were chosen $(150 \mathrm{~km}, 250 \mathrm{~km}$ and $350 \mathrm{~km})$ and simulated on the representative driving cycles from Fig. 4. The weighting factors, determined from the distribution of trip distances within Germany, are 0.5 for the $150 \mathrm{~km}$ trip and 0.25 for the $250 \mathrm{~km}$ and $350 \mathrm{~km}$ trip.

Concerning the scenario without the requirement for long-range capability, two trip distances ( $75 \mathrm{~km}$ and $100 \mathrm{~km}$ ) were chosen and the weighting factors were set to 0.6 and 0.4 , respectively.

\section{Vehicle Simulation Model}

A vehicle simulation model was developed to calculate the energy consumption of variable parametrizations for multiple powertrain concepts. The energy demand and fuel consumption of each vehicle parametrization is used to estimate the ecological impact of the vehicle during operational phase. In order to ensure the comparability of different powertrain concepts, every vehicle parametrization created by the optimization algorithm shares an equal base vehicle body with predefined parameters like rolling resistance coefficient, drag coefficient or base 
mass. Only the structure and dimensioning of powertrain components is adapted to create different parametrizations of a powertrain concept.

For this reason, a generic powertrain model was created that consists of components for the energy converters (Internal Combustion Engine ICE, Electric Machine EM and Generator G), as well as the transmission and the battery. The architecture is shown in Fig. 5. Subdividing the transmission into three parts allows for a convenient differentiation in the model between parallel hybrid powertrain topologies like P2 or P3 configuration.

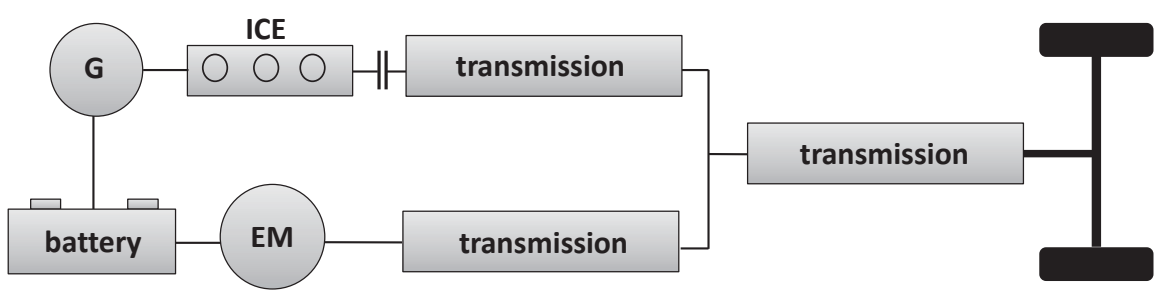

Fig. 5: Architecture of the developed generic powertrain model

Based on this architecture, the following powertrain concepts listed in

Table 3 are defined and investigated within this framework.

Table 3: Powertrain concepts investigated within this framework

\begin{tabular}{|l|l|}
\hline ICEV-O & Conventional Vehicle with petrol engine \\
\hline mHEV & Mild hybrid vehicle in P2 configuration \\
\hline BEV & Battery electric vehicle with fixed gear ration transmission \\
\hline BEV-2eG & Battery electric vehicle with 2-speed transmission \\
\hline SREV & Series hybrid with range extender \\
\hline PHEV(P2) & Parallel plug-in hybrid in P2 configuration \\
\hline PHEV(P3) & Parallel plug-in hybrid in P3 configuration \\
\hline PHEV(P3-2eG) & $\begin{array}{l}\text { Parallel plug-in hybrid in P3 configuration with an additional dedicated } \\
\text { 2-speed transmission for the EM }\end{array}$ \\
\hline
\end{tabular}

As described in Section 2, a number of powertrain parameters are being optimized in order to identify the optimal parametrization for each powertrain concept that minimizes the REI. This approach allows for an unbiased comparison of the technological potential of the different 
powertrain concepts. Therefore, methods are required to scale the components of the powertrain based on the optimization parameters. Alongside the efficiencies of the components, which mainly determine the fuel consumption and energy demand of the vehicle during the operational phase, the masses of the powertrain components have a significant impact on the total $\mathrm{CO}_{2}$ emissions of the vehicle. They both increase the consumption of the vehicle due to an increased vehicle weight and determine the emissions during production and end-of-life phases.

The EM is scaled by means of its power. Different powers require adapted efficiency maps as well as an adapted mass which is especially relevant for the estimation of the ecological impact during production. In our approach, we define a reference permanent magnet synchronous electrical machine and apply a torque based scaling of the efficiency map to match it with the respective power

$$
\frac{T_{\mathrm{EM}}}{T_{\text {ref }}}=\frac{P_{\mathrm{EM}}}{P_{\text {ref }}}
$$

where $T$ and $P$ denote the torque and power of the reference and scaled machine, respectively. The mass of the EM as a function of power is estimated with the help of a nonlinear regression curve derived from literature data [6, 7]. This method is likewise applied to other components of the powertrain, e.g. the inverter.

Concerning the ICE, a different approach is chosen. To avoid the necessity of scaling the complex characteristics of an ICE efficiency map, a database of several engines with varying power is created. The optimizing algorithm is thus not choosing the ICE power from a continuous interval, but discrete values according to the engines in the database. Every engine is defined by its brake specific fuel consumption map including the full load characteristics and therefore its power, the type of fuel used and its mass. The mass is again estimated as a function of the ICE power by a regression curve based on collected data.

The design matching of the transmission and the engines has a great influence on vehicle consumption and the capability to fulfill the special design constraints mentioned in Section 2. Therefore, transmission ratios are also being optimized in this framework and the mass of the transmission is calculated based on an empirical correlation as a function of maximum torque, transmission ratio and gear number [8].

The vehicle simulation model is a backwards facing model of the longitudinal dynamics and consists of two processing steps. At first, the traction demand of the vehicle at the wheels required to follow the driving cycle is calculated by means of a driving resistances equation, 
taking drag, rolling resistance, grade resistance and acceleration resistance into account. Subsequently, the operating strategy chooses operating points for the energy converters to meet the traction demand at every time step that ultimately determine an overall vehicle consumption. Losses in the powertrain components are modelled with constant efficiencies for the transmissions and battery and efficiency maps for the ICE and the EM. Even though the efficiencies of the transmission and the battery are generally also dependent on the operating point or external influencing factors, the sensitivity of the REI with regard to transmission and battery efficiency is rather low and thus not considered.

An ECMS operating strategy is applied to choose locally optimal operating points for the energy converters with respect to consumption, thus deciding on gear shifting as well as the torque split between ICE and EM. The cost function $J$ of the ECMS which is being minimized in every time step specifies an equivalent fuel mass flow rate of electrical and petrochemical energy $[2,3]$. The cost ratio of electrical and petrochemical energy is defined by the equivalent cost factor $s$.

$$
J=b_{\mathrm{e}} P_{\mathrm{ICE}}+s \frac{1}{L H V} \frac{1}{\eta_{\mathrm{EM}} \eta_{\text {batt }}} P_{\mathrm{EM}}
$$

In Equation $8, b_{e}$ denotes the brake specific fuel consumption, $L H V$ the lower heating value, and $\eta$ the efficiency. For powertrain concepts with only a single energy converter, the number of degrees of freedom reduces to one since the torque can not be split upon two energy converters. Apart from that, the operating strategy can be applied to all powertrain concepts in the same fashion, which ensures the comparability of the consumption estimation of different powertrain concepts. This definition of an operating strategy also allows for additional operating modes of parallel hybrid powertrain concepts, e.g. boosting or load point shifting.

The exact value of the cost factor $s$ is chosen for every parametrization individually in an inner iteration loop. The mild hybrid, since it does not have the option of external charging, needs to operate in charge sustaining mode. The cost factor of the plug-in hybrids, on the other hand, is set such that the whole usable electric energy of the battery is depleted at the end of a driving cycle, thus assuming an optimal user behaviour in terms of regular charging after each trip.

\section{Results}

Within this section, the results of the overall optimization framework for the chosen scenarios are presented. Firstly, the analysis for reference year 2018 is presented. For this reference 
year, a scenario with and without the requirement of long-range capability (350 km and $100 \mathrm{~km}$ range, respectively) are compared. Secondly, the results for the scenario with the requirement for long-range capability in the reference year 2030 are summarized.

\subsection{Scenario without the requirement for long-range capability $(100 \mathrm{~km})$ in the refer- ence year 2018}

The scenario without the requirement for long-range capability in 2018 is defined by the following set of parameters shown in Table 4.

Table 4: Parameter set for the scenario without the requirement for long-range capability in 2018.

\begin{tabular}{|l|l|}
\hline Total range & $100 \mathrm{~km}$ \\
\hline Specific emissions of energy production & $578 \mathrm{~g} \mathrm{CO}_{2} / \mathrm{kWh}$ \\
\hline Specific energy of the battery & $109 \mathrm{Wh} / \mathrm{kg}$ \\
\hline Total vehicle mileage & $158.000 \mathrm{~km}$ \\
\hline
\end{tabular}

The parametrizations of the powertrain concepts for this scenario, determined by the optimzation framework, are summarized in Table 5. The parameters correspond to the powertrain concept parametrizations that results in a minimal REI for the investigated scenario. The battery capacities of the BEV are relatively low $(\sim 22 \mathrm{kWh})$ but sufficient for the moderate range requirement. Comparing the required electrical power of the single-speed and twospeed BEV shows that the two-speed transmission resolves conflicting design goals between maximum vehicle speed and necessary starting torque, resulting in less required power for the BEV-2eG. The reduced demand of installed power and the potential to choose the operating gear lead to a reduction of electric consumption for the BEV-2eG of $\sim 4 \%$ compared to the BEV. 
Table 5: Powertrain parameters of the different concepts determined by the optimization framework for the scenario without the requirement for long-range capability in 2018.

\begin{tabular}{|l|r|r|l|l|l|l|l|}
\hline Concept & $\begin{array}{l}\text { Power } \\
\text { ICE in kW }\end{array}$ & $\begin{array}{l}\text { Power } \\
\text { EDM in kW }\end{array}$ & $\begin{array}{l}\text { Battery } \\
\text { capacity in kWh }\end{array}$ & $\begin{array}{l}\text { Total mass } \\
\text { in kg }\end{array}$ & $\begin{array}{l}\text { El. consumption } \\
\text { in kWh/100km }\end{array}$ & $\begin{array}{l}\text { Fuel consumption } \\
\text { in l/100km }\end{array}$ & $\begin{array}{l}\text { Max. Torque } \\
\text { required in Nm }\end{array}$ \\
\hline ICEV-O & 96 & 0 & 0 & 1110 & 0,0 & 5,0 & 1657 \\
\hline mHEV & 96 & 21 & 2 & 1157 & 0,0 & 4,2 & 1728 \\
\hline BEV & 0 & 145 & 22,4 & 1274 & 17,9 & 0,0 & 1903 \\
\hline BEV(2eG) & 0 & 82 & 21,6 & 1260 & 17,2 & 0,0 & 1882 \\
\hline sREV & 50 & 145 & 16,9 & 1298 & 16,2 & 0,6 & 1939 \\
\hline PHEV(P2) & 50 & 34 & 14,5 & 1284 & 14,0 & 0,9 & 1918 \\
\hline PHEV(P3) & 50 & 55 & 16,2 & 1285 & 15,6 & 0,5 & 1920 \\
\hline PHEV(P3-2eG) & 50 & 39 & 15 & 1270 & 14,4 & 0,7 & 1897 \\
\hline
\end{tabular}

The PHEV concepts show a large difference in maximum EM power between the series (SREV) and parallel topologies. The series hybrid is limited to use the power of the electric traction machine to satisfy the design constraints, whereas the parallel hybrids can transmit the combined power of the EM and the ICE to the wheels. The PHEV in P3 topology requires the highest amount of electrical power among the parallel PHEVs, since the purely electric city driving operation (design constraint), as well as reaching the required maximum speed, must be enabled with a single-speed transmission. The hybrids in P2 and P3-2eG topology, on the other hand, can use the multispeed transmission for the EM.

For the PHEV concepts, optimal battery capacities of $\sim 15-17 \mathrm{kWh}$ are identified which satisfy the design constraint of $60 \mathrm{~km}$ electrical range. With this battery capacity, the hybrid concepts are also able to achieve high electrical driving percentages on trips up to $100 \mathrm{~km}$, as shown by the low petrochemical consumption in Table 5. In the case of the series hybrid, it is advantageous to increase the battery capacity in order to reduce the otherwise necessary operation of the range extender, since this leads to disadvantages in the overall electric consumption due to multiple energy conversion steps.

In terms of the total weight of the vehicles, the optimum variants of the conventional and mild hybrid vehicle are the lightest. The BEV and the PHEV have similar weights, as the BEV needs a larger battery and the PHEV has additional installed powertrain components.

The determined REI of the different powertrain concepts are illustrated in Fig. 6. A general result is that the electrification of powertrains is beneficial for the scenario without the requirement for long-range capability, since all electrified powertrains outperform the conventional ICEV. The BEV are well suited for this scenario due to the moderate battery capacities and the generally high efficiency of the powertrain. The two-speed BEV yields the best results for this scenario. 
The parallel PHEV show REI values in between the results of the BEV. The optimization environment optimizes the powertrain concepts such that synergies are used efficiently for the entire system, resulting in dedicated hybrid transmissions (DHT). For example, the parallel PHEV can only meet the high driving requirements using both traction machines. When driving at medium power requirements, the PHEV can operate using the small parametrized EM at a high specific utilization which is beneficial for a high efficiency. In addition, the available electric energy of the battery is distributed by the operating strategy using hybrid functionalities (purely electric or combustion engine driving, load-shift \& boosting).

Further details on the $\mathrm{CO}_{2}$-emissions during the production of the powertrain components are summarized in Fig. 7. The PHEV concepts have the highest number of necessary components for the powertrain. The ICEV and BEV concepts only require their corresponding components. In this scenario without the requirement for long-range capability, the glider, which gathers all components that are not part of the powertrain, has the highest impact. The production of the battery also shows a relevant amount of $\mathrm{CO}_{2}$-emissions. In the case of higher installed battery capacities for the scenarios with the requirement for long-range capability, the emissions during the production of the battery become most relevant. 


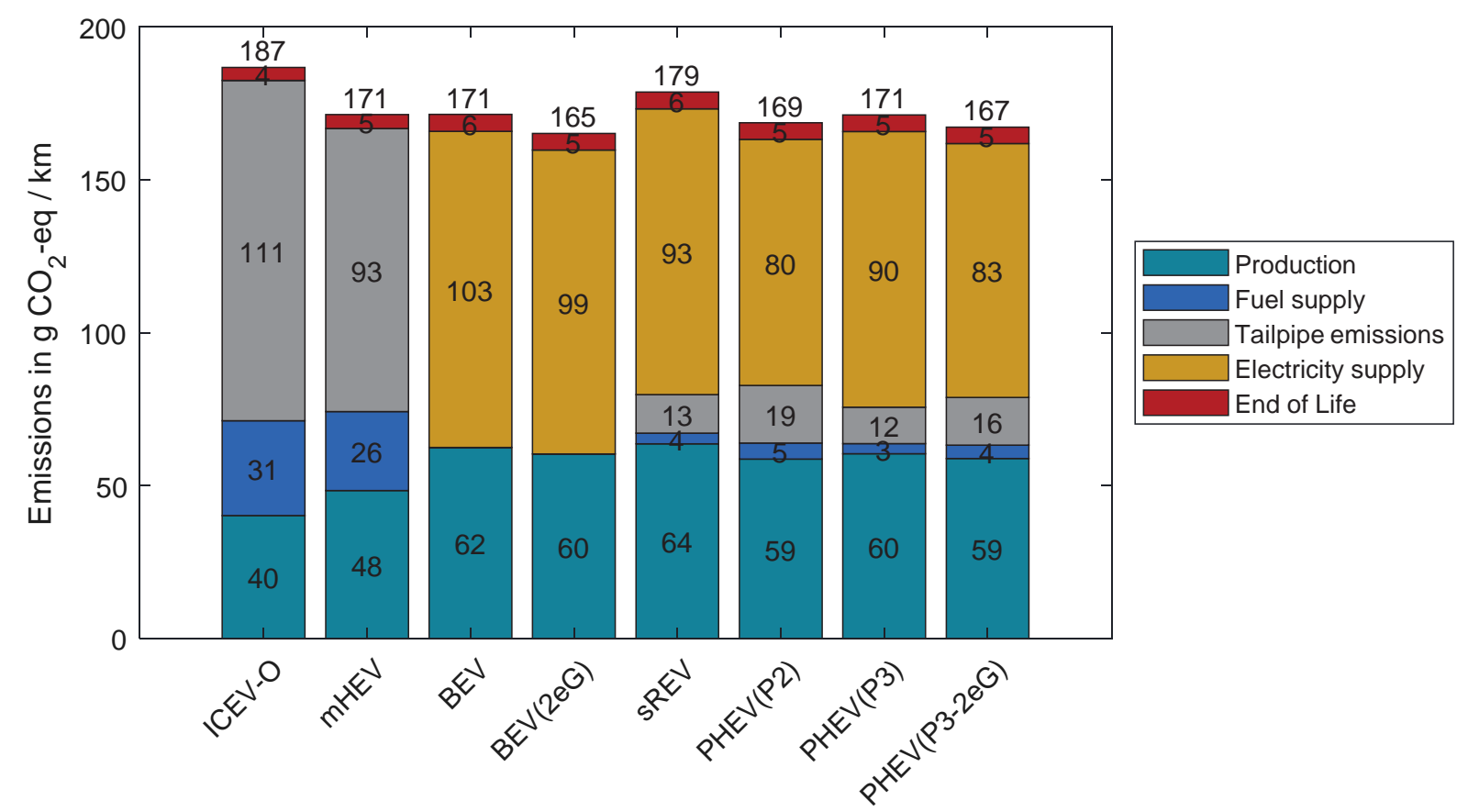

Fig. 6: Identified Real Ecological Impact of the different powertrain concepts for the scenario without the requirement for long-range capability in the reference year 2018.

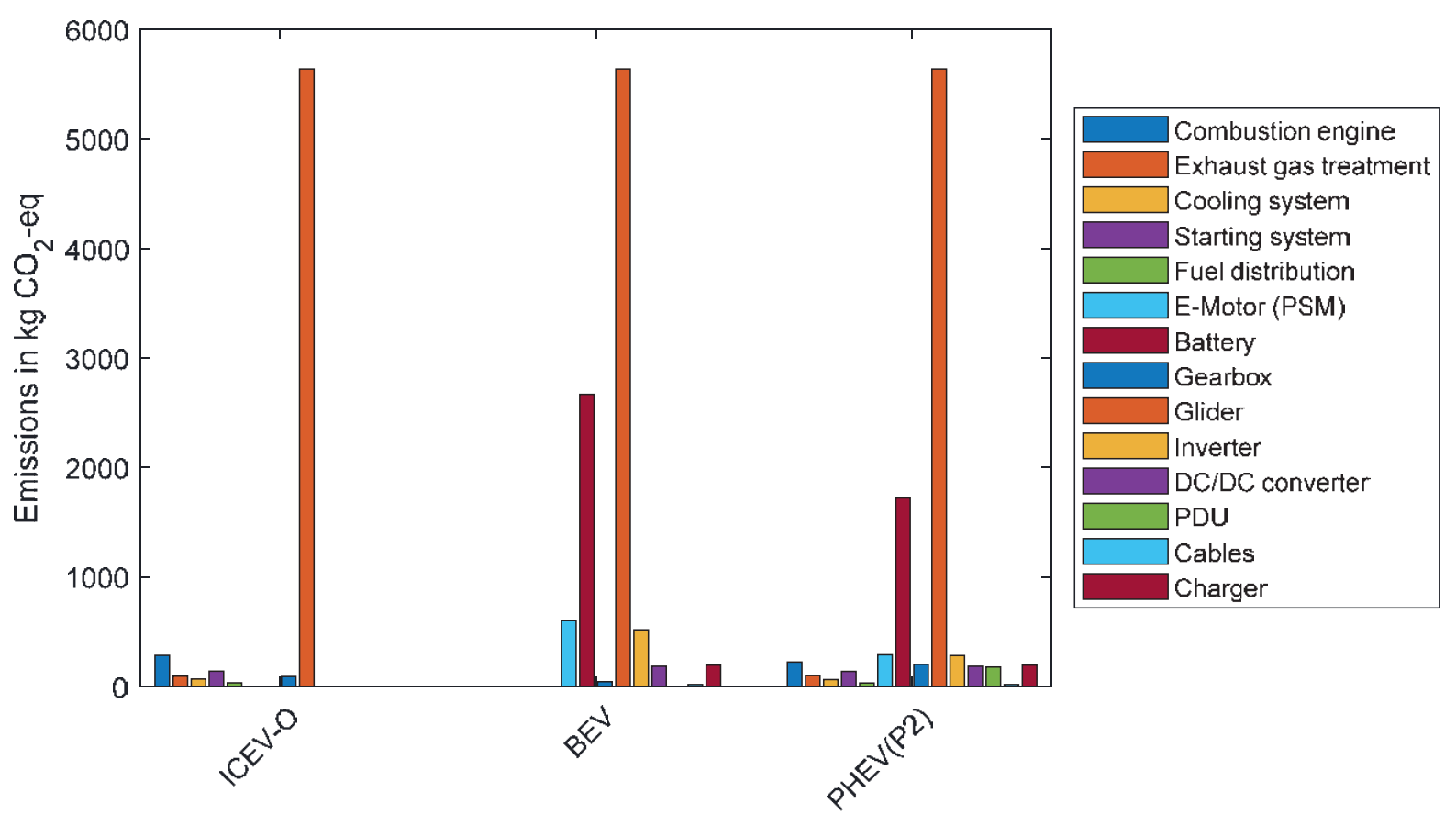

Fig. 7: $\mathrm{CO}_{2}$-emissions in the production of vehicle components for the scenario without the requirement for long-range capability in the reference year 2018. 


\subsection{Scenario with the requirement for long-range capability $(350 \mathrm{~km})$ in the reference year 2018}

The same parameter set of Table 4 is applied to this scenario, except for an increased range requirement of $350 \mathrm{~km}$. The calculated powertrain concept parametrizations, determined by the optimization procedure for the parametrization of the powertrain concepts, are summarized in Table 6. The ICEV-O and the mHEV only show minor deviations from the scenario without the requirement for long-range capability. The differences arise from the fact that the evaluation cycles and their weights between the driving cycles, as described in Section 3, differ in the scenarios with and without the requirement for long-range capability. For the BEV on the other hand, there are major differences compared to the scenario without the requirement for longrange capability. First, the required battery capacity to achieve the total range of the scenario increases significantly. This leads to a high vehicle weight and required maximum torque at the wheels. The single-speed BEV needs a high amount of installed power in order to meet the starting torque and the maximum speed requirements in combination. The two-speed BEV, on the other hand, can meet the requirements with significantly less power due to its transmission.

The series PHEV (SREV) also requires a lot of power due to its single-speed transmission. The optimization identifies an optimal battery capacity of approximately $18 \mathrm{kWh}$ and relies on greater use of the range extender to charge the battery during operation, thus achieving the required range despite lower battery capacity compared to the BEV.

In the case of the parallel PHEV, there are only slight differences in the parametrization in comparison to the scenario without the requirement for long-range capability. Due to longer trip distances, the fuel consumption per $100 \mathrm{~km}$ increases significantly compared to the scenario without the requirement for long-range capability. At the same time, less electric energy is available per distance, which is why the electric consumption per distance is lower. As discussed in Section 4, the operating strategy ensures that the entire available battery capacity is always used. 
Table 6: Powertrain parameters of the different concepts determined by the optimization framework for the scenario without the requirement for long-range capability in 2018.

\begin{tabular}{|l|r|r|l|l|l|l|l|}
\hline Concept & $\begin{array}{l}\text { Power } \\
\text { ICE in kW }\end{array}$ & $\begin{array}{l}\text { Power } \\
\text { EDM in kW }\end{array}$ & $\begin{array}{l}\text { Battery } \\
\text { capacity in kWh }\end{array}$ & $\begin{array}{l}\text { Total mass } \\
\text { in kg }\end{array}$ & $\begin{array}{l}\text { El. consumption } \\
\text { in kWh/100km }\end{array}$ & $\begin{array}{l}\text { Fuel consumption } \\
\text { in l/100km }\end{array}$ & $\begin{array}{l}\text { Max. Torque } \\
\text { required in Nm }\end{array}$ \\
\hline ICEV-O & 96 & 0 & 0 & 1110 & 0,0 & 5,1 & 1657 \\
\hline mHEV & 96 & 22 & 2 & 1160 & 0,0 & 4,2 & 1733 \\
\hline BEV & 0 & 219 & 90,4 & 1910 & 20,9 & 0,0 & 2853 \\
\hline BEV(2eG) & 0 & 95 & 84,1 & 1842 & 19,4 & 0,0 & 2751 \\
\hline sREV & 50 & 206 & 17,5 & 1311 & 7,1 & 3,3 & 1958 \\
\hline PHEV(P2) & 50 & 32 & 14,5 & 1278 & 1283 & 2,7 & 1908 \\
\hline PHEV(P3) & 50 & 49 & 16,3 & 1283 & 6,6 & 1917 \\
\hline PHEV(P3-2eG) & 50 & 33 & 15 & 1266 & 6,1 & 2,6 & 1891 \\
\hline
\end{tabular}

The identified REI over the entire life cycle of the powertrain concepts are shown in Fig. 8. A comparison with the scenario without the requirement for long-range capability reveals significant differences. The BEV concepts show a major increase in their REI values. This is primarily due to the large battery capacities to meet the requirements, which lead to excessive $\mathrm{CO}_{2}$-emissions in production and, secondly, to an increased vehicle weight, which in turn affects the consumption. Since the design requirements have to be covered with the high vehicle weight, there is also a very high electric power required, which contributes to the REI during the component production. The two-speed BEV-2eG, as before, has clear advantages over the single-speed BEV, since the required electrical power and the electric consumption can be reduced significantly ( $\sim 9 \%$ compared to the $\mathrm{BEV}$ ). This also results in a smaller required battery capacity. However, the determined REI shows that BEV concepts are not optimal for a long-range capable vehicle in the reference scenario for 2018.

The parallel PHEV and the mHEV perform best under all considered powertrain concepts. The comparison to the scenario without the requirement for long-range capability shows that the ICE of the PHEV is used much more on the now longer trip distances. This worsens the REI of the parallel PHEV compared to the short-distance scenario. Although the mHEV cannot charge external electric energy, it also has significantly lower production costs than the PHEV, and reaches the best balance with a small margin to the PHEV concepts within this scenario. As before, the P2 topology is slightly advantageous over the P3 topology and the innovative P3-2eG concept performs best for the PHEV concepts with a small margin. 


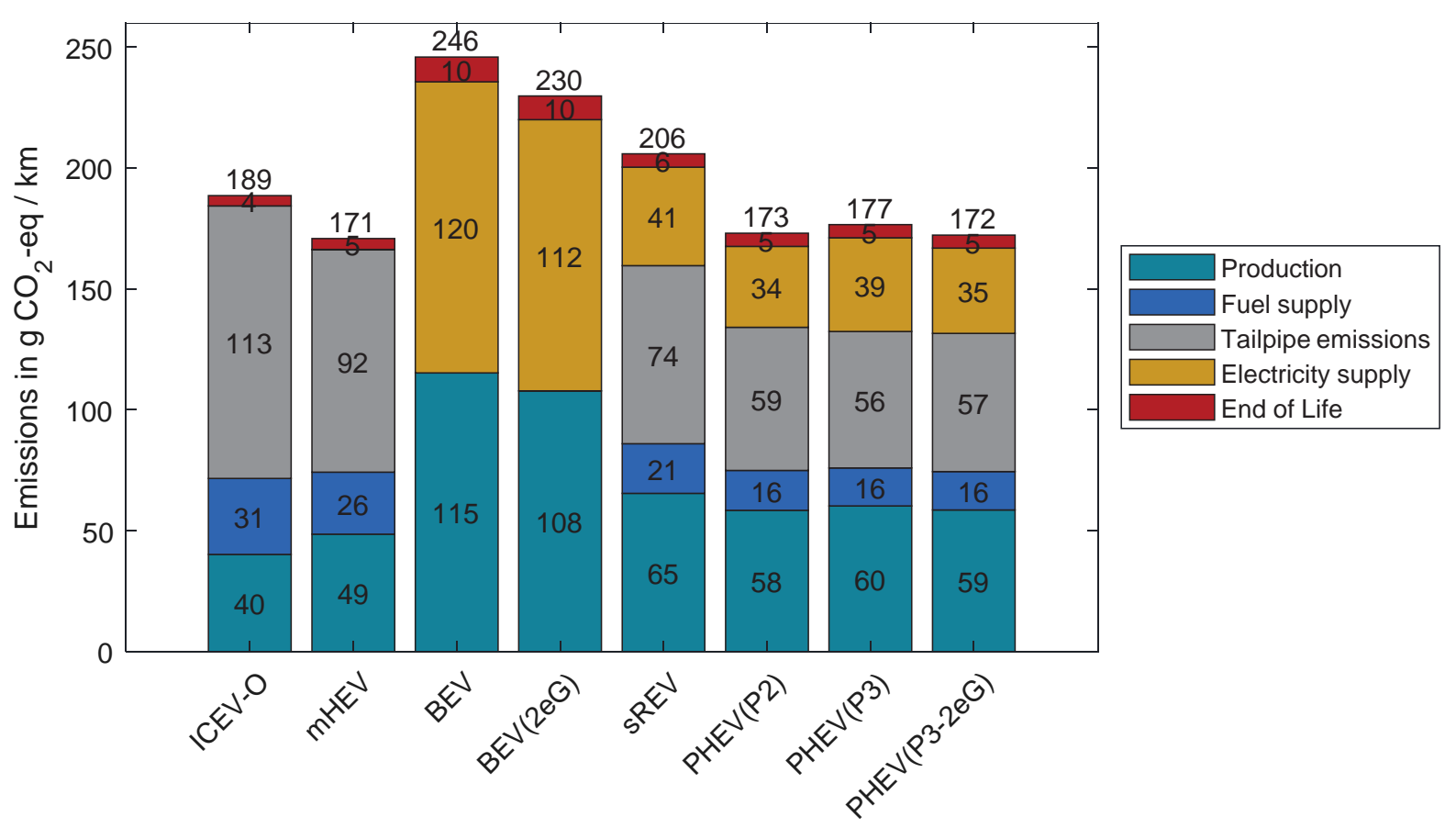

Fig. 8: Identified Real Ecological Impact of the different powertrain concepts for the scenario with the requirement for long-range capability in the reference year 2018.

The operating behaviour of the parallel PHEV in P2 topology on the first driving cycle for a trip distance simulation of $350 \mathrm{~km}$ is shown in Fig. 9. The simulation of operating behaviour is carried out within the optimization framework for many different parametrizations of all powertrain concepts in order to identify the minimal achievable REI. 

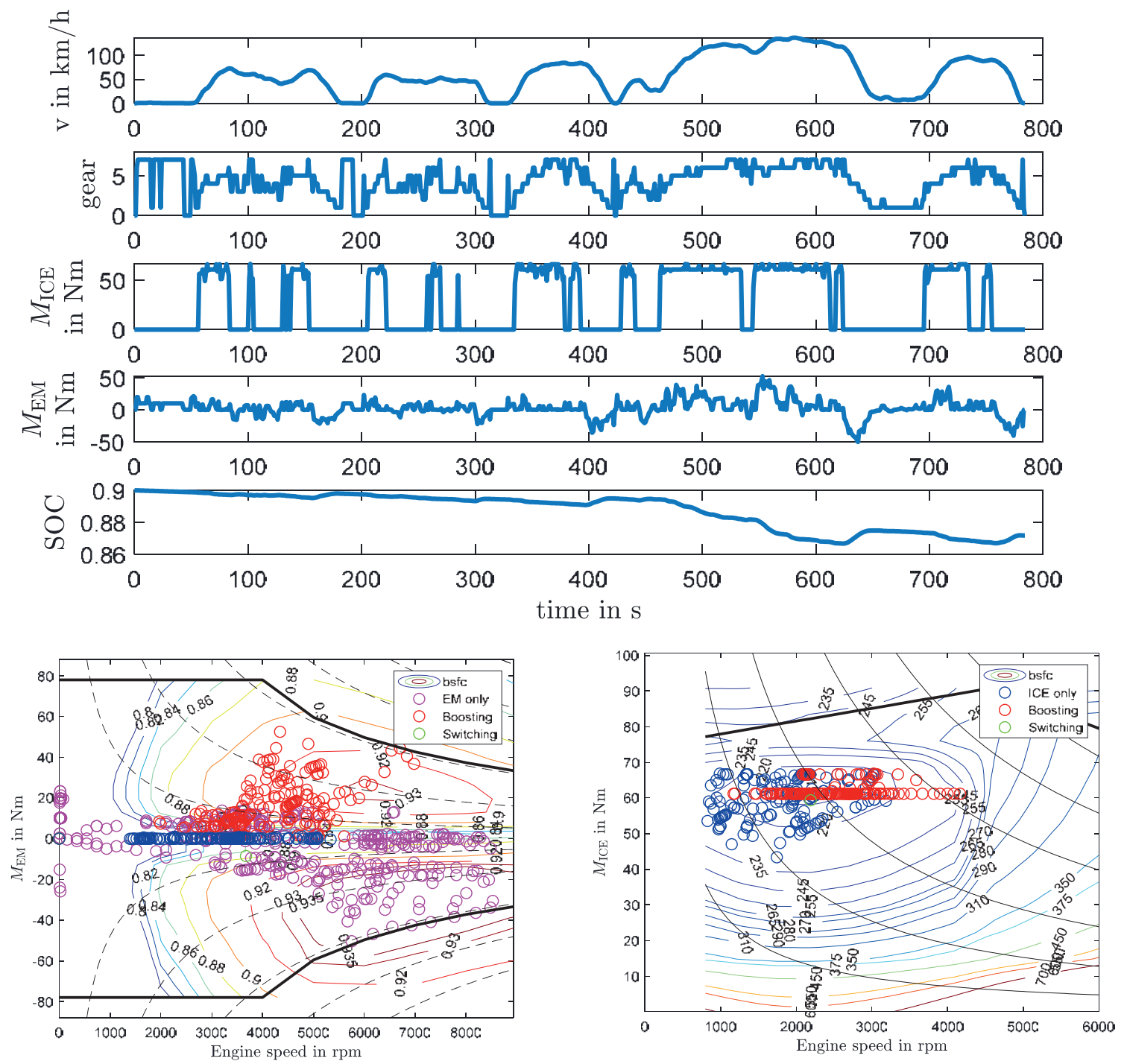

Fig. 9: Exemplary simulation of a parallel PHEV in P2 topology 


\subsection{Scenario with the requirement for long-range capability $(350 \mathrm{~km})$ in the reference year 2030}

The parameter set of this scenario is summarized in

Table 7.

Table 7: Parameter set for the scenario with the requirement for long-range capability in the reference year 2030.

\begin{tabular}{|l|l|}
\hline Total range & $350 \mathrm{~km}$ \\
\hline Specific emissions of energy production & $447 \mathrm{~g} \mathrm{CO}_{2} / \mathrm{kWh}$ \\
\hline Specific energy of the battery & $126 \mathrm{Wh} / \mathrm{kg}$ \\
\hline Total vehicle mileage & $158.000 \mathrm{~km}$ \\
\hline
\end{tabular}

The parameters of the powertrain concepts identified by the optimization environment are shown in Table 8. For the ICEV-O, there is no difference to the reference year 2018, as it does not benefit from the improved specific emissions of the energy production or the higher energy density of the battery, and further improvements of combustion technology or fuel properties have not been taken into account. The mild hybrid benefits only marginally, as the vehicle weight is slightly reduced due to the improved battery technology.

Table 8: Powertrain parameters of the different concepts determined by the optimization framework for the scenario without the requirement for long-range capability in 2018.

\begin{tabular}{|l|r|r|l|l|l|l|l|}
\hline Concept & $\begin{array}{l}\text { Power } \\
\text { ICE in kW }\end{array}$ & $\begin{array}{l}\text { Power } \\
\text { EDM in kW }\end{array}$ & $\begin{array}{l}\text { Battery } \\
\text { capacity in kWh }\end{array}$ & $\begin{array}{l}\text { Total mass } \\
\text { in kg }\end{array}$ & $\begin{array}{l}\text { El. consumption } \\
\text { in } \mathrm{kWh} / 100 \mathrm{~km}\end{array}$ & $\begin{array}{l}\text { Fuel consumption } \\
\text { in l/100km }\end{array}$ & $\begin{array}{l}\text { Max. Torque } \\
\text { required in Nm }\end{array}$ \\
\hline ICEV-O & 96 & 0 & 0 & 1109 & 0,0 & 5,1 & 1657 \\
\hline mHEV & 96 & 20 & 2 & 1155 & 0,0 & 4,2 & 1725 \\
\hline BEV & 0 & 199 & 87,5 & 1772 & 20,3 & 0,0 & 2647 \\
\hline BEV(2eG) & 0 & 92 & 82,3 & 1721 & 19,0 & 0,0 & 2571 \\
\hline sREV & 50 & 192 & 36,3 & 1438 & 14,6 & 1,3 & 2147 \\
\hline PHEV(P2) & 50 & 33 & 14,5 & 1259 & 5,8 & 2,7 & 1880 \\
\hline PHEV(P3) & 50 & 49 & 16,3 & 1262 & 6,7 & 2,5 & 1886 \\
\hline PHEV(P3-2eG) & 50 & 32 & 15 & 1246 & 5,9 & 2,6 & 1862 \\
\hline
\end{tabular}


Significant changes, however, arise in the BEV parametrizations. Thanks to the improved battery technology, these vehicles can operate with significantly reduced weight compared to the scenario with the requirement for long-range capability from 2018. The lower weight also results in less electric power necessary to meet all design requirements, which also reduces the specific electric consumption. The improved efficiency can also further reduce the required battery capacity compared to the reference year 2018 .

The series hybrid gives further interesting results. The battery capacity of the vehicle is increased compared to the reference year 2018, which reduces the need to provide electric energy by the range extender and reduces fuel consumption.

In the case of parallel hybrids, there are no significant changes in the parametrizations. The parallel operation with the ICE is significantly more efficient than the series mode of operation of the SREV and it is still worthwhile for the parallel hybrids to use this operation mode extensively in the reference year 2030. Due to the higher energy density of the battery, a little less weight leads to slightly reduced consumptions compared to the scenario with the requirement for long-range capability in 2018.

The calculated REI of the powertrain concepts are shown in Fig. 10 for scenario with the requirement for long-range capability in the reference year 2030. The BEV improve the most compared to the scenario of 2018 and the two-speed BEV achieves a comparable REI level to the ICEV-O. Despite the technological developments taken into account, the REI of the BEV concepts still suffer from high emissions that are caused by the production of the vehicles. 


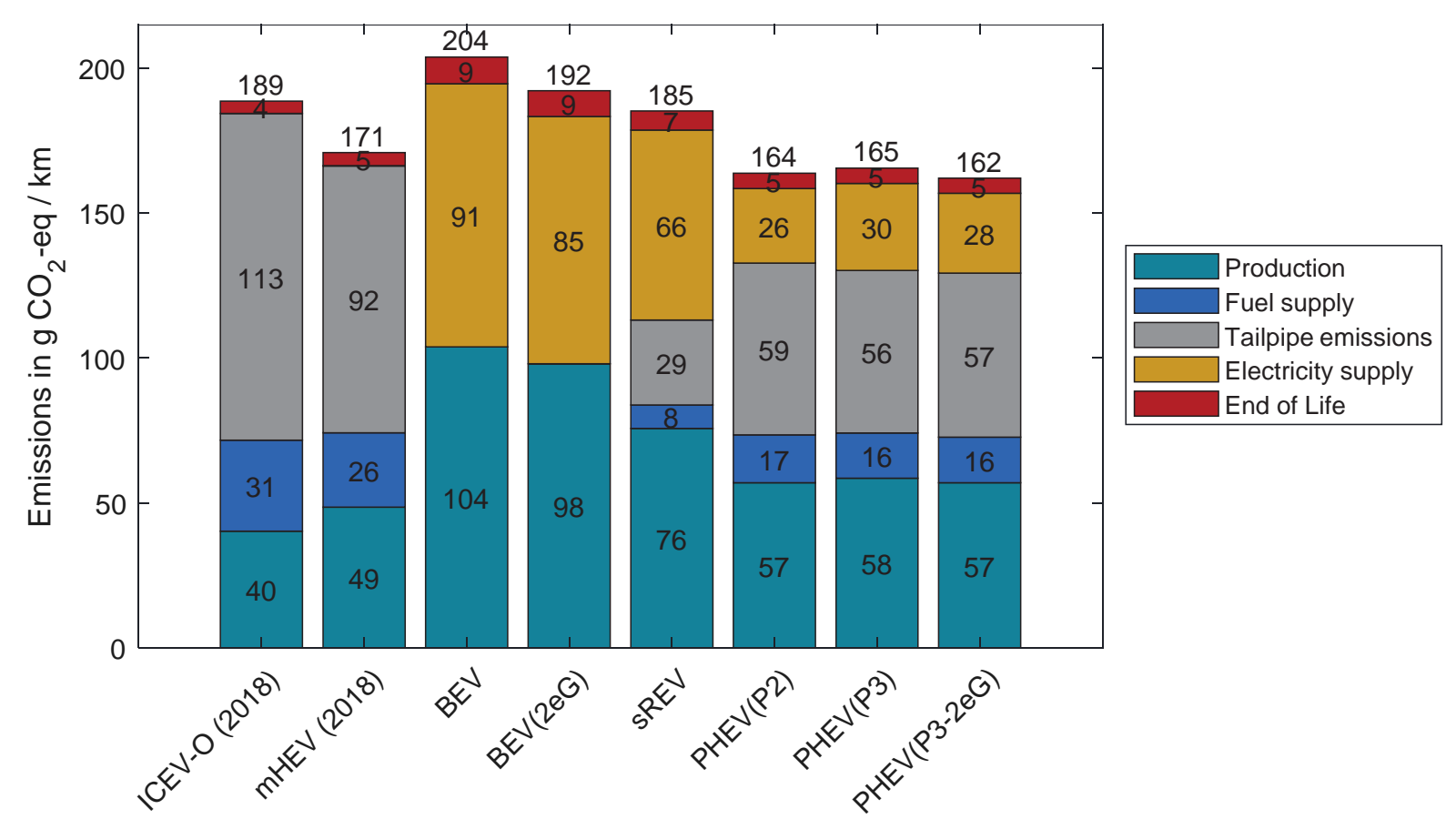

Fig. 10: Identified Real Ecological Impact of the different powertrain concepts for the scenario with the requirement for long-range capability in the reference year 2030. Technological progression in ICE technology was not considered.

As in the previous scenarios, the series hybrid has disadvantages compared to the parallel topologies. The parallel PHEV perform best in the reference year 2030 and show a significant improvement, which is mainly due to the improved electricity mix. As in the previous results, the P2 topology shows advantages over the P3 topology, due to the multispeed transmission that can be used by the electric machine. The P3-2eG topology performs best among the parallel hybrids due to the variety of operating options.

Overall, the parallel PHEV have the greatest potential to reduce the REI for the scenario with the requirement for long-range capability in the reference year 2030. Despite the moderate battery capacity, a purely electric, locally emission-free city driving and a relatively high total electrical driving percentage are achieved, since the shortest simulated trip distance with the highest electrical driving percentage has the largest weighting factor in the consumption assessment.

\section{Summary and Conlusions}

This work presents an optimization framework for the comparative evaluation of the Real Ecological Impact (REI) of different powertrain concepts. We show that in order to establish an unbiased comparison, multiple criteria need to be considered. 
First of all, the greenhouse gas emissions of the vehicle need to be considered over the whole life cycle, including production, operational and end-of-life phases.

Secondly, the consumption of the vehicles needs to be determined by means of real driving data. Therefore, we applied a cycle synthesis method based on real driving profiles. By that, we are able to compress big fleet data sets into computationally efficient but representative driving cycles.

Moreover, the parametrization of powertrain concepts has a significant effect on both the emissions in the production phase and the consumption during the operational phase. The comparison of existing production vehicles cannot characterize the actual potential of a powertrain concept to reduce the REI since different design goals were considered in the development of these vehicles. We created a framework that explicitly optimizes the parametrization of the powertrain concepts based on real driving data to reduce the REI of the vehicles and thus, enables to assess the actual potential of the respective powertrain concepts.

We analyzed the REI of a conventional vehicle, multiple hybrid electric vehicles with diverse powertrain topologies, and two purely electric powertrain concepts. The results are shown for scenarios with and without the requirement for long-range capability of $100 \mathrm{~km}$ and $350 \mathrm{~km}$ range, respectively. The scenario with the requirement for long-range capability is additionally investigated for a predicted scenario parameter set in the year 2030.

We identified a significant impact of the chosen scenario on the assessment of the powertrain concepts. Battery electric vehicles, today and especially in the future, have a good potential to reduce the REI in the scenario without the requirement for long-range capability, but they perform much worse in the scenarios with the requirement for long-range capability where the required large battery capacity leads to a high energy consumption due to an increased vehicle weight and particularly to high emissions during the production phase. Even for the year 2030, the predicted increase in battery density and the decreased specific emissions of energy production cannot compensate for this.

The best results of the scenario with the requirement for long-range capability are achieved by the parallel plug-in hybrids and the mild hybrid. Interestingly and in contrast to current production vehicles, all of the powertrains are optimized towards DHT that use the advantages of both energy converters and also the synergy of the combined use of the energy converters. The ICE and EM can be dimensioned smaller which reduces the emissions in the production phase. The parallel plug-in hybrids are also benefiting from the improved scenario parameters with 
regards to electric energy production and energy storage. Moreover, the parallel plug-in hybrids perform well on the scenario without the requirement for long-range capability, since the powertrains are optimized towards a high percentage of electrical driving.

In general, parallel plug-in hybrid concepts are identified to robustly achieve a good ecological potential for all investigated scenarios. Concerning the scenario with the requirement for longrange capability, the parallel PHEV perform best, even when the scenario of 2030 is considered. Still, the prediction of future REI for different powertrain concepts is linked to significant uncertainties because of the high sensitivity to external factors. The developed optimization framework allows for a proper comparison of the ecological potential of different powertrain concepts under changing boundary conditions on an equal evaluation basis and is therefore suited to be used in an agile manner, adapting to the latest predictions of boundary conditions.

\section{Acknowledgements}

We thank our partners in the publicly funded project FahrKLang. This article would not have been possible without their contribution to the project. In addition to the partner institutes (IWAR) and (VKM) at TU Darmstadt, the DLR Institute of Transport Research (DLR VF) is to be mentioned.

\section{Supported by:}

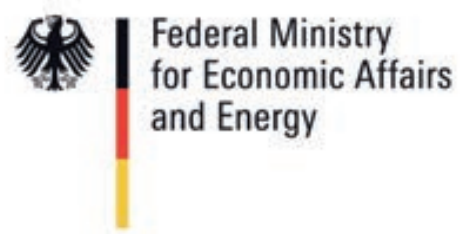

on the basis of a decision

by the German Bundestag 


\section{References}

[1] A. Esser, M. Zeller, S. Foulard, and S. Rinderknecht, "Stochastic Synthesis of Representative and Multidimensional Driving Cycles," in WCX World Congress Experience, 2018.

[2] G. Paganelli, S. Delprat, T. M. Guerra, J. Rimaux, and J. J. Santin, "Equivalent consumption minimization strategy for parallel hybrid powertrains," in IEEE 55th Vehicular Technology Conference, Birmingham, AL, USA, 2002, pp. 2076-2081.

[3] L. Serrao, S. Onori, and G. Rizzoni, "ECMS as a realization of Pontryagin's minimum principle for HEV control," in American Control Conference, St. Louis, MO, USA, 2009, pp. 3964-3969.

[4] Ingo Totzke et al., "Sichere Intelligente Mobilität: Deliverable D41.1," 2010. [Online] Available: https://www.eict.de/projekte/\#project-19.

[5] infas institut für angewandte sozialwissenschaft gmbh and Deutsches Zentrum für Luftund raumfahrt e.V. - Institut für Verkehrsforschung, Eds., "Mobilität in Deutschland 2008," FE-Nr. 70.801/2006, 2010.

[6] A. Nordelöf, E. Grunditz, A.-M. Tillman, T. Thiringer, and M. Alatalo, "A scalable life cycle inventory of an electrical automotive traction machine-Part I: Design and composition," (en), Int J Life Cycle Assess, vol. 23, no. 1, pp. 55-69, 2018.

[7] A. Balazs, Optimierte Auslegung von Hybridantriebsträngen unter realen Fahrbedingungen. Fakultät für Maschinenwesen, RWTH Aachen, Dissertation, 2015.

[8] Mitschke, M., Wallentowitz, H., Dynamik der Kraftfahrzeuge: Springer, 2014. 